\title{
Radiation
}

ISSN 0909-0495

Editor: G. Ice

\section{X-ray beam monitor made by thin-film CVD single-crystal diamond}

\author{
Marco Marinelli, E. Milani, G. Prestopino, C. Verona, G. Verona-Rinati, M. \\ Angelone, M. Pillon, V. Kachkanov, N. Tartoni, M. Benetti, D. Cannatà and \\ F. Di Pietrantonio
}

J. Synchrotron Rad. (2012). 19, 1015-1020

Copyright (C) International Union of Crystallography

Author(s) of this paper may load this reprint on their own web site or institutional repository provided that this cover page is retained. Republication of this article or its storage in electronic databases other than as specified above is not permitted without prior permission in writing from the IUCr.

For further information see http://journals.iucr.org/services/authorrights.html

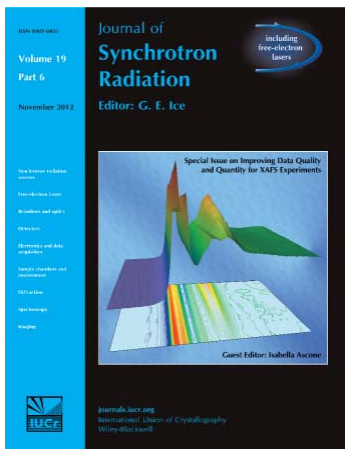

Synchrotron radiation research is rapidly expanding with many new sources of radiation being created globally. Synchrotron radiation plays a leading role in pure science and in emerging technologies. The Journal of Synchrotron Radiation provides comprehensive coverage of the entire field of synchrotron radiation research including instrumentation, theory, computing and scientific applications in areas such as biology, nanoscience and materials science. Rapid publication ensures an up-to-date information resource for scientists and engineers in the field.

Crystallography Journals Online is available from journals.iucr.org 
Journal of

Synchrotron

Radiation

ISSN 0909-0495

Received 29 May 2012

Accepted 5 September 2012

(C) 2012 International Union of Crystallography Printed in Singapore - all rights reserved

\section{X-ray beam monitor made by thin-film CVD single-crystal diamond}

\author{
Marco Marinelli, ${ }^{a}$ E. Milani, ${ }^{a}$ G. Prestopino, ${ }^{a}$ C. Verona, ${ }^{a} *$ G. Verona-Rinati, ${ }^{a}$ \\ M. Angelone, ${ }^{b}$ M. Pillon, ${ }^{b}$ V. Kachkanov, ${ }^{c}$ N. Tartoni, ${ }^{c}$ M. Benetti, ${ }^{\text {d }}$ D. Cannatàd \\ and F. Di Pietrantonio ${ }^{d}$
}

\begin{abstract}
anip. di Ingegneria Industriale, Università di Roma 'Tor Vergata', Via del Politecnico 1, I-00133 Roma, Italy, ${ }^{\mathbf{b}}$ Associazione EURATOM-ENEA sulla Fusione, Via E. Fermi 45, I-00044 Frascati (Roma), Italy, ' Diamond Light Source, Harwell Science and Innovation Campus, Chilton, Didcot, Oxfordshire OX11 0DE, UK, and 'CNR, Istituto di Acustica 'O.M. Corbino', Via del Fosso del Cavaliere 100, I-00133 Roma, Italy. E-mail: claudio.verona@uniroma2.it
\end{abstract}

\begin{abstract}
A novel beam position monitor, operated at zero bias voltage, based on highquality chemical-vapor-deposition single-crystal Schottky diamond for use under intense synchrotron X-ray beams was fabricated and tested. The total thickness of the diamond thin-film beam monitor is about $60 \mu \mathrm{m}$. The diamond beam monitor was inserted in the B16 beamline of the Diamond Light Source synchrotron in Harwell (UK). The device was characterized under monochromatic high-flux X-ray beams from 6 to $20 \mathrm{keV}$ and a micro-focused $10 \mathrm{keV}$ beam with a spot size of approximately $2 \mu \mathrm{m} \times 3 \mu \mathrm{m}$ square. Time response, linearity and position sensitivity were investigated. Device response uniformity was measured by a raster scan of the diamond surface with the micro-focused beam. Transmissivity and spectral responsivity versus beam energy were also measured, showing excellent performance of the new thin-film single-crystal diamond beam monitor.
\end{abstract}

Keywords: CVD diamond; beam position monitor; X-ray detector; Schottky diode.

\section{Introduction}

Synchrotron light is an important tool available for many types of research applications which span from biology to solid state physics (Als-Nielsen \& McMorrow, 2011). For many experiments it is of paramount importance that the collected data are normalized to the impinging beam intensity. The latter can be measured by a beam intensity monitor (BIM) usually located upstream of the sample. The BIM must fulfil many requests such as excellent linearity as a function of the photon flux, well characterized behavior versus photon energy and negligible interaction with the beam in order to reduce beam absorption and scattering. The need for low X-ray energy has increased with time so the BIM must also ensure good response and performances down to the lowest available $\mathrm{X}$-ray energy. Furthermore, since the most recent synchrotrons produce high-intensity beams, the BIM must also withstand high X-ray radiation dose.

Up to now the most used BIMs are ionization chambers (ICs) (Bosshard et al., 1992). ICs have been used since the early days of radiation and synchrotron studies. However, ICs have some drawbacks such as large dimensions and, when operated under intense beams, non-linear response owing to space charge regime. The latter effect can be lessened by filling the chamber with helium. Ionization chambers with large dimensions are not helpful when the experiment needs little beam perturbation such as in low-angle-scattering experiments or when space constraints prevent their use. Compact and scarcely invasive beam monitors which also fulfil the request to operate under intense $\mathrm{X}$-ray beams, to have a linear response and to introduce negligible perturbation of the beam flux are in principle feasible by using solid state detectors. Conventional sensors based on semiconductor photodetectors, mostly silicon, generally involve the complete absorption of the incoming radiation (Gauthier et al., 1995). Among the several candidates, thanks to its low atomic number, high thermal conductivity, high electric resistivity and excellent charge transport properties, diamond is an ideal material for the fabrication of radiation-hard on-line X-ray beam monitors. In particular, owing to a low absorption coefficient for X-rays over the energy range $5-25 \mathrm{keV}$ (Field, 1979), diamond is a very promising semi-transparent material for in situ beam monitoring (Bergonzo et al., 2006; Morse et al., 2006; Bohon et al., 2010). However, detector-grade natural diamonds are extremely rare and expensive, high-pressure high-temperature (HPHT) diamonds are strongly impaired by concentration levels of different impurities (Pace \& De Sio, 2003) and polycrystalline chemical vapor deposition (CVD) 
diamonds have poor response times and spatial homogeneity (McKeag \& Jackman, 1998; Marczewska et al., 2007). As a result, unstable responsivity and polarization affected the realisation of satisfactory diamond-based detectors. Polarization effects, owing to trapping of carriers, are responsible for time variations of charge collection efficiency in CVD diamond (Manfredotti et al., 2006). The homoepitaxial CVD growth offers also a technology for reproducibly producing high-quality single-crystal diamond film on low-cost diamond substrates (Angelone et al., 2010; Liao \& Koide, 2006).

Unlike the detectors reported by Bergonzo et al. (2006), Morse et al. (2006) and Bohon et al. (2010), a novel beam position monitor, operating at zero bias voltage, made of a very thin film of synthetic single-crystal diamond and suitable for use under intense synchrotron X-ray beams has been fabricated. The detector is based on a multi-layered Schottky diode structure grown on top of a low-quality HPHT diamond substrate. In order to allow a resolution beam position sensitivity, a multiple-electrodes geometry was used. In this paper the results obtained with the new X-ray beam monitor during its testing performed at the Diamond Light Source synchrotron are presented and discussed.

\section{Diamond beam monitor fabrication process}

The diamond beam monitor (DBM) is based on a multilayered Schottky diode structure. Using a microwave plasma enhanced CVD apparatus a conductive boron-doped diamond and a high-purity intrinsic single-crystal diamond layer were homoepitaxially grown in sequence on a mechanically polished HPHT diamond substrate. The thickness of both CVD diamond films was about $1 \mu \mathrm{m}$. The size of the substrate was $4 \mathrm{~mm} \times 4 \mathrm{~mm} \times 0.3 \mathrm{~mm}$. The intrinsic and B-doped layers were deposited using two different CVD reactors in order to avoid any boron contamination. The intrinsic diamond layer, which operates as the detecting region, has been oxidized after growth by isothermal annealing at $773 \mathrm{~K}$ for $1 \mathrm{~h}$ in air, in order to remove the $\mathrm{H}_{2}$ surface conductive layer. After the growth of the CVD diamond films the thickness of the HPHT diamond substrate was reduced by anisotropic reactive ion etching (RIE) of the back of the substrate. To this purpose the diamond substrate was cleaned and introduced into the vacuum chamber on a cooled sample holder and pumped down to the background pressure of $10^{-6}$ torr. A gas mixture composed of $25 \% \mathrm{CF}_{4}$ in $\mathrm{O}_{2}$ was fluxed in the chamber with a flow rate of $20 \mathrm{sccm}$, reaching a pressure of $15 \mathrm{mtorr}$, and a power of $200 \mathrm{~W}$ was applied, obtaining an etch rate of $3.8 \mu \mathrm{m} \mathrm{h}^{-1}$. At the end of the process the total thickness of the diamond thin-film beam monitor was about $60 \mu \mathrm{m}$ (see Fig. 1b). Figs. 1( $a$ ) and 1(c) show SEM images of the surfaces of the CVD intrinsic diamond and the etched HPHT diamond substrate, respectively. The grown CVD diamond surface is very smooth without superficial structural defects. On the other hand the surface profile of the diamond substrate after the etching process is rough with holes up to $10 \mu \mathrm{m}$ deep (see Fig. 1c).

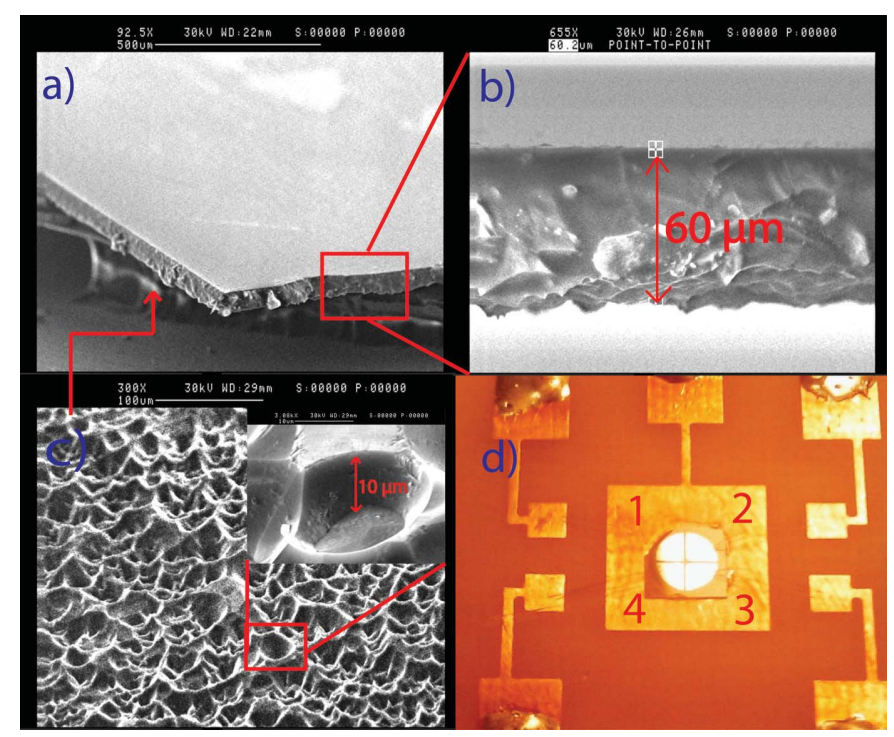

Figure 1

SEM images of (a) CVD diamond film, (b) a side view of the DBM, (c) the back of the HPHT substrate after RIE treatment. (d) Photograph of the DBM as assembled on a printed circuit board (the micro-soldering on four electrodes is clearly visible).

Finally, a $50 \mathrm{~nm}$-thick rectifying low-atomic-number aluminium circular electrode ( $3 \mathrm{~mm}$ in diameter) was thermally evaporated on the CVD intrinsic diamond surface, while annealed silver paint was used in order to provide an ohmic contact with the B-doped layer. As reported by Almaviva et al. (2010), such a structure acts as a sandwich-type metal/intrinsic/ p-doped diamond Schottky barrier diode. It must be noted that the particular detector structure used is able to operate without any external bias voltage as a result of its internal junction electric field (Almaviva et al., 2010). By dividing the electrical contact into four sections with an inter-electrode distance of $150 \mu \mathrm{m}$ by a standard photolithography technique and removing aluminium by wet etching, a four-electrode beam position monitor was fabricated. A photograph of the device wire bonded on its printed circuit (PC) board is shown in Fig. 1(d).

\section{Experimental set-up}

Testing of the DBM was performed at beamline B16 of Diamond Light Source (DLS), a versatile beamline designed to test optics, detectors and other instrumentation. DLS operated with an electron beam current of $250 \mathrm{~mA}$ and in topup mode so that the change in the beam current was within $1 \%$ during the entire experiment. The intensity of the X-ray beam was monitored with an ionization chamber located upstream of the diamond detector. The monochromatic beam is produced by a Si (111) double-crystal monochromator or a $\mathrm{Ru} / \mathrm{B}_{4} \mathrm{C}$ double-multilayer monochromator (Sawhney et al., 2011). In the experiment the monochromatic beam in the spectral region from $6 \mathrm{keV}$ to $20 \mathrm{keV}$ was used. The beam spot size was $500 \mu \mathrm{m} \times 500 \mu \mathrm{m}$. The spot size was determined by means of slits. For the operation at low energy a tube filled 
with helium was used to minimize the air path so reducing the $\mathrm{X}$-ray absorption.

A compound refractive lens was installed to obtain a microfocused spot size in order to scan the surface of the detector. In this case the beam energy was fixed at $10 \mathrm{keV}$. The parabolic shape provides bi-dimensional focusing. The dimensions of the focal spot were measured as $3 \mu \mathrm{m}$ FWHM in the horizontal direction and $2 \mu \mathrm{m}$ FWHM in the vertical direction.

The X-ray interaction in diamond creates electron-hole pairs that drift in the material. The four signals generated by the four electrodes were read out by a FEMTO variable-gain low-noise current amplifier, model DLPCA-200 (FEMTO Messtechnik GmbH, Germany; http://www.femto.de/). The detector was mounted on a PC board with a hole to allow the beam to impinge on the detector and placed on a highprecision translational stage that enabled the surface of the detector to be scanned.

The data acquisition of the beamline was used for the steady state and slowly varying signals, while an oscilloscope was used to acquire the transient of the photocurrent. In order to measure the photon flux, expressed in photons $\mathrm{s}^{-1}$, a calibrated silicon detector was used and mounted on the translational stage.

\section{Results and discussions}

The electrical characterization of the DBM was performed at room temperature by measuring the current-voltage $(I-V)$ characteristics using a Keithley 6517A pico-amperemeter. Each $I-V$ characteristic was obtained by applying a voltage to each aluminium electrode while the backing contact p-type diamond layer was earthed. The $I-V$ curves of four electrodes in reverse and forward polarizations are reported in Fig. 2. Similar curves were obtained, having the typical shape for a diode with a rectification ratio of $5 \times 10^{3}$ at $\pm 4 \mathrm{~V}$. Extremely low dark currents of the order of $10^{-15} \mathrm{~A}$ were found at $0 \mathrm{~V}$ bias, slightly increasing at higher reverse biases. The device can only operate in the reverse-bias mode because when operating in the forward bias mode the photocurrent is masked by the dark current (Almaviva et al., 2009; Imura et al., 2009).

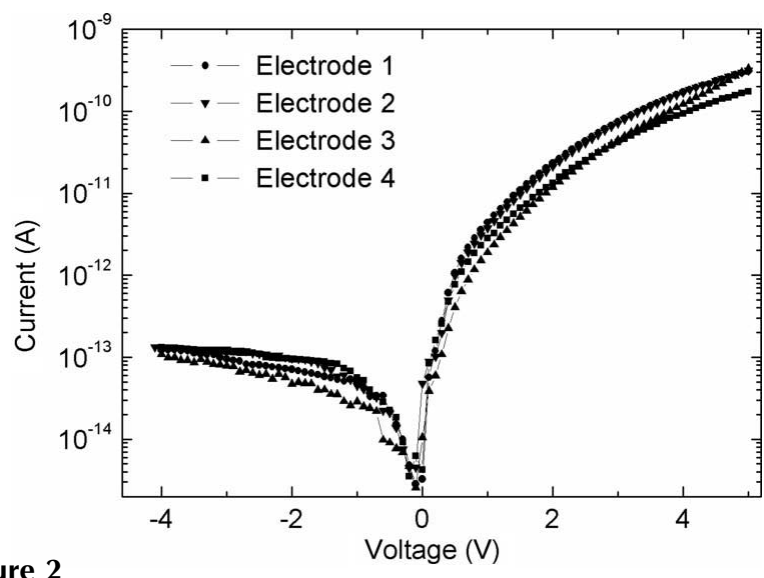

Figure 2

$I-V$ curves of the four electrodes.

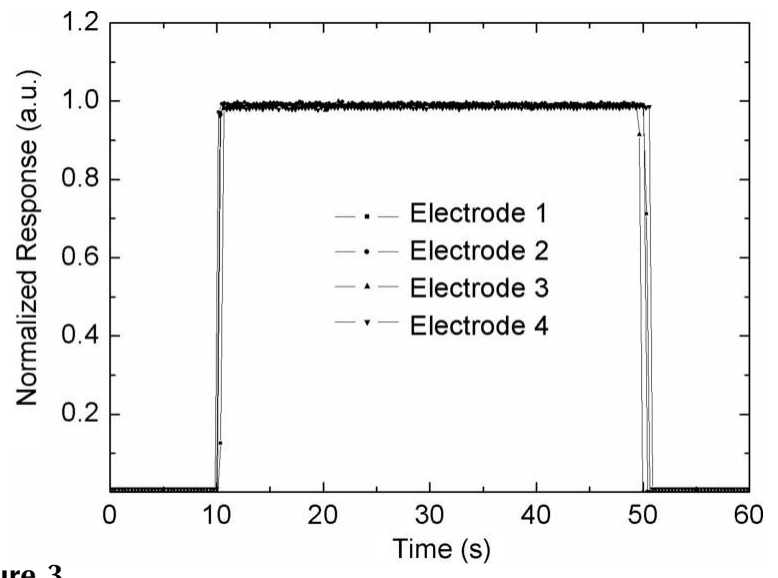

Figure 3

Time response of the four electrodes under an $8 \mathrm{keV}$ X-ray photon beam.

The DBM was aligned with respect to the photon beam at a fixed photon energy of $8 \mathrm{keV}$ searching for the maximum response in each electrode. In Fig. 3 the normalized photocurrent signal of four electrodes as a function of time is reported by opening and closing a mechanical shutter during the acquisition. The DBM was operated in unbiased mode. The response is reproducible and undesired effects such as persistent photocurrent, overshoot or slow component of the response were not observed. The $300 \mathrm{~ms}$ rise and fall times do not represent the real device response time being fully accounted by the acquisition time constant. In order to better evaluate the capability of the detector to follow rapid beam fluctuations, in Fig. 4 the photocurrent acquired by the oscilloscope during X-ray irradiation chopped at $1 \mathrm{kHz}$ is reported. The preamplifier was set in full bandwidth mode. Rise and decay times of the photoresponse are about $60 \mu \mathrm{s}$, corresponding to the full opening time of the aperture of the mechanical chopper.

The linearity of the DBM has also been investigated and the results are reported in Fig. 5. The beam intensity was varied moving two blades which partially intercept the beam. For the linearity tests two runs were performed at each fixed flux, one illuminating the diamond detector and the other illuminating the silicon detector. Owing to both the maximum available

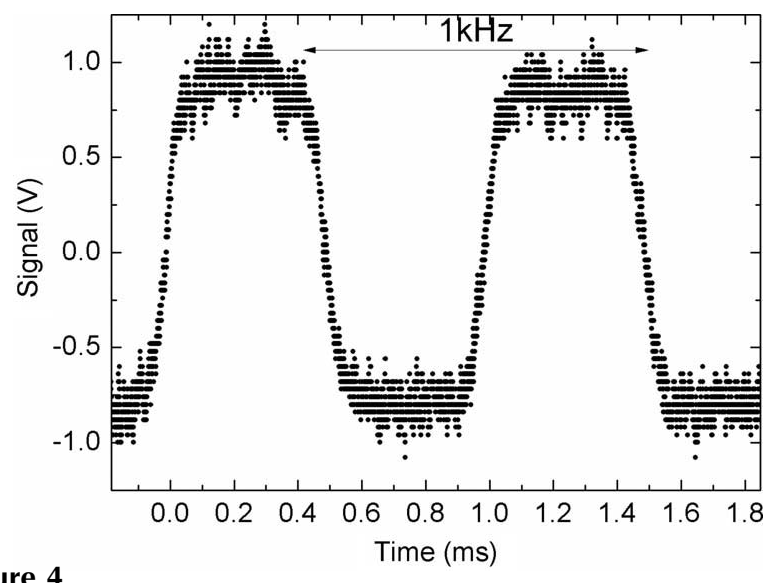

Figure 4

Temporal response of the DBM under an $8 \mathrm{keV}$ X-ray beam chopped at $1 \mathrm{kHz}$. 


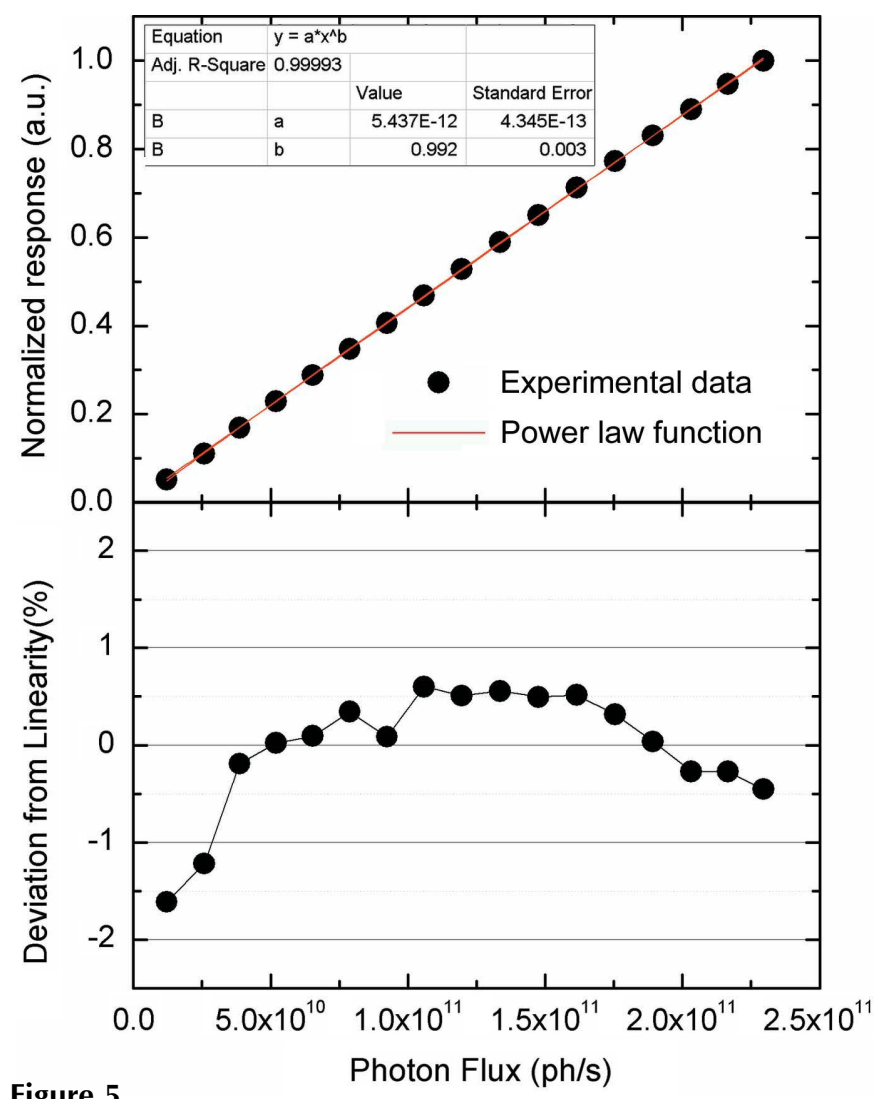

Figure 5

(a) Linearity of the DBM (the red straight line shows the result of fitting the power law) and $(b)$ the percentage deviation from linearity.

photon flux and the limit posed by the silicon dark current, only one decade in the linearity range was investigated. The DBM shows a good linear behavior and the linearity index was found to be $b=0.9917$, as given by fitting the experimental data with the power law function $y=a x^{b}$.

The spectral response of the DBM at different bias voltages is shown in Fig. 6, where the responsivities (A/W) of the four electrodes are reported as a function of the energy in the range $6-20 \mathrm{keV}$. The absolute responsivities versus X-ray energy were obtained by comparing the current measured by

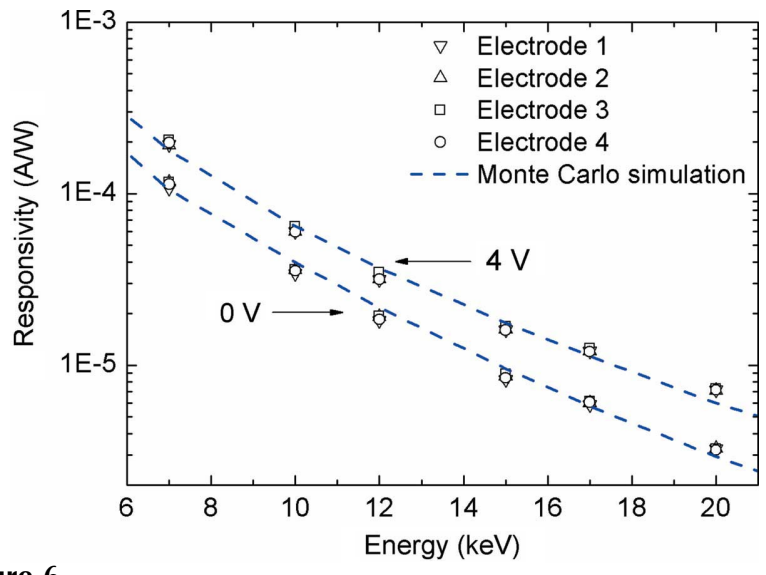

Figure 6

Experimental and calculated responsivity of the DBM at $0 \mathrm{~V}$ and $4 \mathrm{~V}$ bias. Dashed lines are Monte Carlo simulation curves.
DBM with the photon flux measured by the calibrated silicon detector. A high repeatability in spectral responsivity can be observed. The variation of the mean value of the responsivity measured by the four aluminium electrodes is lower than $0.8 \%$ in the whole energy range investigated. The experimental data were compared with a simulation curve obtained by a threedimensional Monte Carlo simulation using the MCNP-X code (see http://mcnp-green.lanl.gov/ for information about $M C N P-5$ radiation transport code). Such responsivity was calculated by taking into account the structure of the DBM detector. The effect owing to the metal contact was included by using $50 \mathrm{~nm}$ for the evaporated $\mathrm{Al}$ contact thickness. The only free parameter in the simulation is therefore the depth of the diamond active layer. The results of the Monte Carlo simulation, reported in Fig. 6 as dashed lines, are in good agreement with the experimental data. This was possible by using a value of $0.5 \mu \mathrm{m}$ and about $1 \mu \mathrm{m}$ for the active diamond layer thickness under $0 \mathrm{~V}$ and $4 \mathrm{~V}$ bias, respectively. The last calculated thickness is approximately the intrinsic diamond film thickness thus demonstrating that the sensing layer of the diamond detector is almost fully depleted at $4 \mathrm{~V}$ bias.

To achieve an absolute calibration, the absorption in the diamond and therefore the spectrum of the absorbed X-rays must be known. The X-ray transmission curve of the DBM detector as a function of the incident photon energy in the 6$20 \mathrm{keV}$ range is shown in Fig. 7. The experimental points were obtained by comparison of the currents measured by the calibrated silicon detector $\left(I_{\mathrm{Si}}\right)$ without and with diamond placed in front of it $\left(I_{\mathrm{S} / \text { Diam }}\right)$, and applying $I_{\mathrm{S} / \text { Diam }} / I_{\mathrm{Si}}$. The experimental data are in good agreement with the calculated curve for $60 \mu \mathrm{m}$-thick diamond using the attenuation data available from NIST Center for X-ray Optics (see http:// www.cxro.lbl.gov/optical_constants/filter2.html for information on filter transmission). In this energy region, diamond absorbs much less than $20 \%$ of the beam, making the DBM ideal for X-ray beam monitoring with low attenuation of X-ray photons.

The homogeneity of the response of the DBM was studied by mapping the diamond surface with a two-dimensional raster scan of a $2 \mu \mathrm{m} \times 3 \mu \mathrm{m}$ square micro-focused X-ray beam. The pitch of the scan was $50 \mu \mathrm{m}$ in both $x$ and $y$

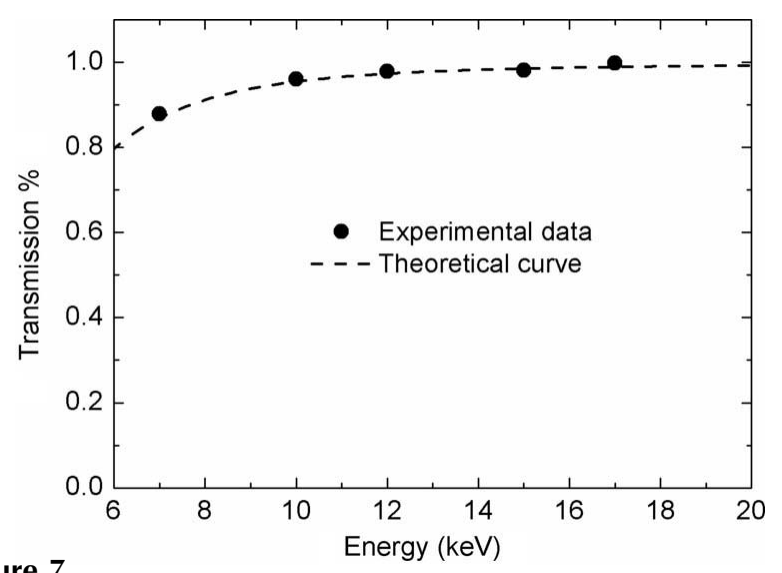

Figure 7

Experimental and calculated transmission of the DBM. 


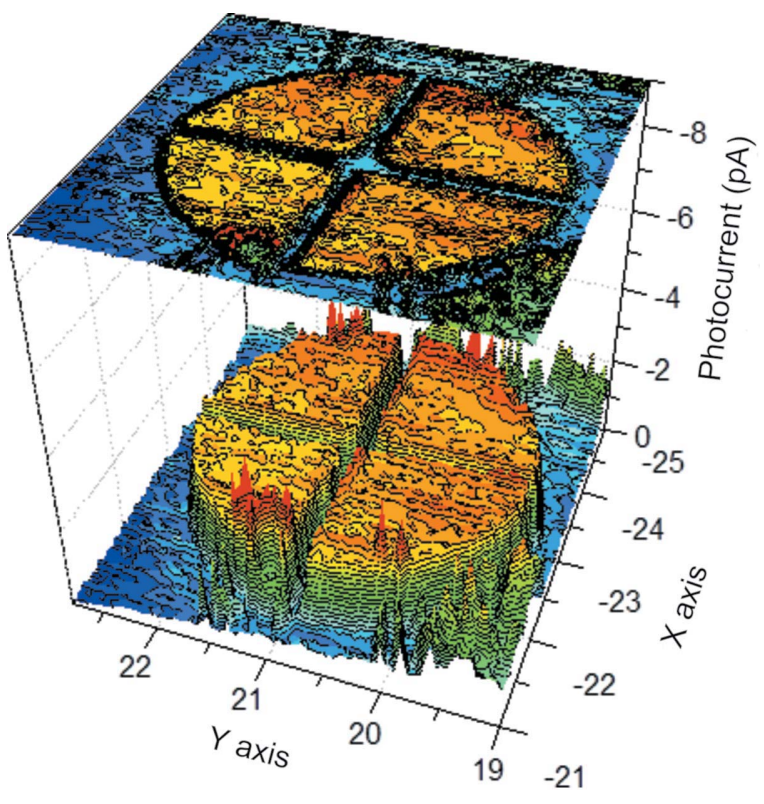

Figure 8

Three-dimensional map and contour plot of the DBM response measured by a raster scan of the DBM surface with a $10 \mathrm{keV}$ micro-focused X-ray beam.

directions. The current from each of the four aluminium electrodes was measured during an overnight exposure. A three-dimensional map and the relative contour plot of the measured currents is displayed in Fig. 8. The device shows for each electrode a maximum fluctuation of about $5 \%$ from the mean value of the relative electrode output currents, while the variation in the mean value between different electrodes is only about $0.5 \%$ which is very similar to the $0.8 \%$ observed using a $500 \mu \mathrm{m}$ beam spot. Such $5 \%$ point-to-point fluctuation of the response across the surface is compatible with the electronic noise (about $100 \mathrm{fA}$ ) during the measurements. Since the sensitive volume is the depleted region underneath the electrodes, the active region of the DBM is defined by the footprint of the Schottky metal contact. Hot spots around the Al wire bonds are also clearly visible in the figure as radial lines. The wire bonds from electrodes generate a weak signal response probably due to photoelectron currents.

The one-dimensional profile of the current measured by scanning in both horizontal and vertical directions the four aluminium electrodes with steps of $10 \mu \mathrm{m}$ is displayed in Figs. 9(a) (vertical) and 9(b) (horizontal). The black and red dotted curves show the behavior of the photocurrent in a region close to the edge of the electrodes at 0 and $4 \mathrm{~V}$, respectively. A very uniform response and a very sharp reduction in the signal are observed in the $150 \mu \mathrm{m}$ gaps separating the electrodes. The broadening at the edge of the electrodes is smaller than the size of the scanning step $(10 \mu \mathrm{m})$. As observed in our previous work (Ciancaglioni et al., 2011), where a similar detector with multi-strip structures was tested under a microfocused X-ray beam, a strip broadening of $4 \mu \mathrm{m}$ was measured, very close to the intrinsic size of the microfocused X-ray beam (about $3 \mu \mathrm{m}$ ). Based on this observation it might be possible to achieve a position sensi-

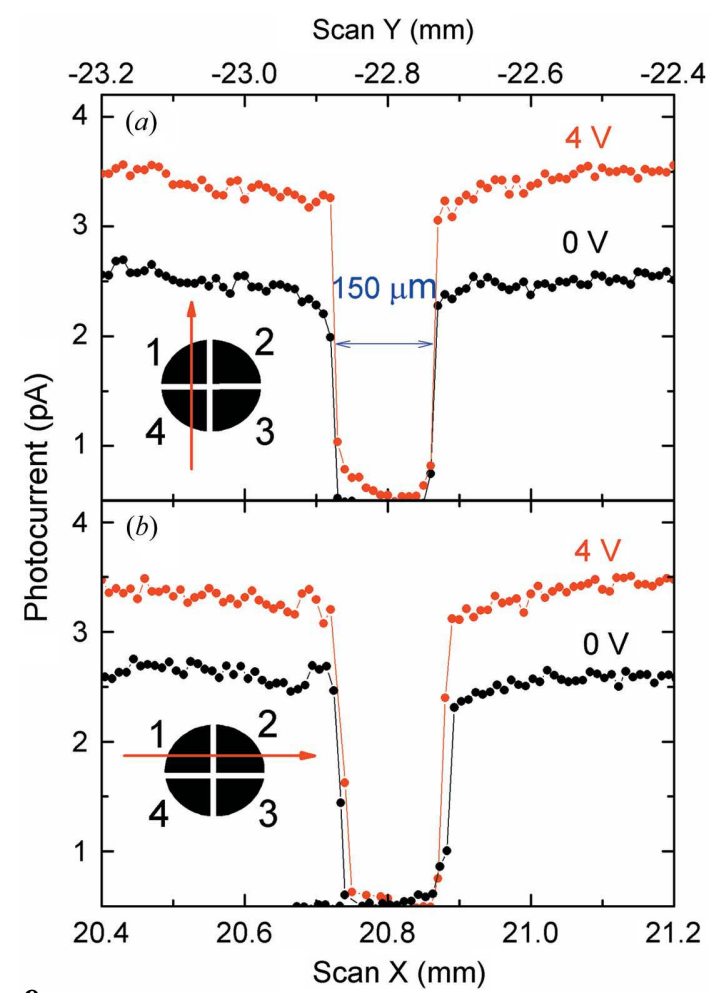

Figure 9

One-dimensional scan between inter-electrodes of the DBM measured in both $(a)$ vertical $(Y)$ and $(b)$ horizontal $(X)$ directions.

tivity of the order of $1 \mu \mathrm{m}$ by decreasing the gap separating the electrodes.

\section{Conclusion}

A single-crystal diamond detector has been successfully fabricated and tested as an X-ray beam monitor at the Diamond Light Source monochromatic synchrotron X-ray beamline. The detector, operating in unbiased mode, consists of a sandwich-type metal/intrinsic/p-doped Schottky barrier diode structure grown on top of a low-quality HPHT singlecrystal diamond substrate. Four metal contacts were deposited on top of the active diamond layer. For optimizing its transmission properties at energies greater than $6 \mathrm{keV}$, the thickness of the whole detector has been minimized by the RIE process applied to the HPHT substrate until a total thickness of about $60 \mu \mathrm{m}$ was obtained. In addition, the structure of the proposed device, having a $\sim 1 \mu \mathrm{m}$-thick active region only, would allow extremely thin free-standing diamond beam monitors to be fabricated, maintaining, in principle, the same radiation detection properties.

The DBM allows position and profile measurements of synchrotron X-ray light sources, together with a low attenuation of X-ray photons $(<20 \%$ at $6 \mathrm{keV})$ of the beam, thus allowing real-time monitoring of the beam with good position sensitivity. Moreover, an excellent linear behavior versus photon flux, good short time stability and a high repeatability in spectral responsivity was observed. The responsivity variation among the four electrodes is lower than $0.8 \%$ over the whole energy range investigated. Finally, local 
point-to-point uniformity of the responsivity across the surface shows a variation of about $5 \%$.

\section{References}

Almaviva, S., Marinelli, M., Milani, E., Prestopino, G., Tucciarone, A., Verona, C., Verona-Rinati, G., Angelone, M., Pillon, M., Dolbnya, I., Sawhney, K. \& Tartoni, N. (2010). J. Appl. Phys. 107, 014511.

Almaviva, S., Marinelli, M., Milani, E., Prestopino, G., Tucciarone, A., Verona-Rinati, G., Angelone, M. \& Pillon, M. (2009). Diamond Relat. Mater. 18, 101-105.

Als-Nielsen, J. \& McMorrow, D. (2011). Elements of Modern X-ray Physics. New York: John Wiley.

Angelone, M., Pillon, M., Marinelli, M., Milani, E., Prestopino, G., Verona, G., Verona-Rinati, C., Coffey, I., Murari, A. \& Tartoni, N. (2010). Nucl. Instrum. Methods Phys. Res. A, 623, 726-730.

Bergonzo, P., Tromson, D. \& Mer, C. (2006). J. Synchrotron Rad. 13, 151-158.

Bohon, J., Muller, E. \& Smedley, J. (2010). J. Synchrotron Rad. 17, 711-718.

Bosshard, R., Kahn, R. \& Fourme, R. (1992). Rev. Sci. Instrum. 63, 810.
Ciancaglioni, I., Marinelli, M., Milani, E., Prestopino, G., Verona, C., Verona-Rinati, G., Angelone, M., Pillon, M., Dolbnya, I., Sawhney, K. \& Tartoni, N. (2011). Europhys. Lett. 94, 28004.

Field, J. E. (1979). Properties of Diamond. London: Academic.

Gauthier, C., Goujon, G., Feite, S., Moguiline, M., Braichovich, L., Brookes, N. B. \& Goulon, J. (1995). Physica B, 208-209, 232-234.

Imura, M., Liao, M., Alvarez, J. \& Koide, Y. (2009). Diamond Relat. Mater. 18, 296-298.

Liao, M. \& Koide, Y. (2006). Appl. Phys. Lett. 89, 113509.

McKeag, R. D. \& Jackman, R. B. (1998). Diamond Relat. Mater. 7, 513-518.

Manfredotti, C., Lo Giudice, A., Vittone, E., Fizzotti, F., Garino, Y. \& Pace, E. (2006). Diamond Relat. Mater. 15, 1467-1471.

Marczewska, B., Kupriyanov, I., Pal'yanov, Yu., Nowak, T., Olko, P., Rębisz, M. \& Waligórski, M. P. R. (2007). Diamond Relat. Mater. 16, 191-195.

Morse, J., Salomé, M. \& Barrett, R. (2006). Ind. Diamond Rev. 66, 34. Pace, E. \& De Sio, A. (2003). Nucl. Instrum. Methods Phys. Res. A, 514, 93-99.

Sawhney, K. J. S., Dolbnya, I. P., Scott, S. M., Tiwari, M. K., Preece, G. M., Alcock, S. G. \& Malandain, A. W. (2011). Proc. SPIE, 8139, 813908. 